\title{
A NORMAL NON-CAYLEY-INVARIANT GRAPH FOR THE ELEMENTARY ABELIAN GROUP OF ORDER 64
}

\author{
GORDON F. ROYLE
}

\author{
(Received 31 August 2007; accepted 8 April 2008) \\ Communicated by Martin W. Liebeck \\ Dedicated to Cheryl Praeger for her sixtieth birthday
}

\begin{abstract}
We exhibit an interesting Cayley graph $X$ of the elementary abelian group $Z_{2}^{6}$ with the property that $\operatorname{Aut}(X)$ contains two regular subgroups, exactly one of which is normal. This demonstrates the existence of two subsets of $Z_{2}^{6}$ that yield isomorphic Cayley graphs, even though the two subsets are not equivalent under the automorphism group of $Z_{2}^{6}$.
\end{abstract}

2000 Mathematics subject classification: 05C25.

Keywords and phrases: Cayley graph, CI-graph, strongly regular graph.

\section{Introduction}

Given group $G$ and a subset $C \subseteq G$, called the connection set, we define the Cayley digraph $\operatorname{Cay}(G, C)$ to be the directed graph with vertex set equal to $G$ and arc-set consisting of all ordered pairs of the form $(g, c g)$ where $c \in C$. It is well known that the automorphism group of $\operatorname{Cay}(G, C)$ contains a regular subgroup isomorphic to $G$, and in fact this condition characterizes Cayley (di)graphs (see, for example, [2]). If we insist that $1 \notin C$, then $\operatorname{Cay}(G, C)$ has no loops, and if we further insist that $C=C^{-1}$, then $\operatorname{Cay}(G, C)$ is undirected. In this paper, both conditions will apply and we will consider only undirected and loopless Cayley graphs. It is clear that, if $\theta \in \operatorname{Aut}(G)$, then

$$
\operatorname{Cay}(G, C) \cong \operatorname{Cay}\left(G, C^{\theta}\right),
$$

but it is well known that the converse is not true, in that two Cayley graphs of $G$ might be isomorphic even though their connection sets are not equivalent under $\operatorname{Aut}(G)$. A Cayley graph $X$ of a group $G$ is said to be a Cayley-invariant graph (CI-graph) of $G$ if every isomorphism between $X$ and another Cayley graph of $G$ is induced by an

(c) 2009 Australian Mathematical Society 1446-7887/09 \$A2.00+0.00 
automorphism of $G$. A graph may be a CI-graph of one group, but not a CI-graph of a different group, so it is essential to specify the group in question. A group $G$ is said to be a Cayley-invariant group (CI-group) if all Cayley graphs of $G$ are CI-graphs of $G$. There has been considerable research into determining which graphs are CI-graphs, which groups are CI-groups, and the directed analogues of these questions. This work is beautifully surveyed by Li [5], who presents all the main results and outlines the many remaining open questions in this area. One useful tool is the observation that whether a graph is a CI-graph or not can be determined from its automorphism group (that is, without direct reference to the putative isomorphic graph).

THEOREM 1. A graph $X$ is a CI-graph for a group $G$ if all regular subgroups of $\operatorname{Aut}(X)$ isomorphic to $G$ are conjugate in $\operatorname{Aut}(X)$.

One of the open questions highlighted by $\mathrm{Li}$ is the problem of characterizing normal non-CI-graphs, that is, non-CI-graphs for a group $G$ whose automorphism group contains a normal regular subgroup isomorphic to $G$. According to $\mathrm{Li}$, normal nonCI-graphs 'seem to be very rare'. In the current paper we contribute to this study by exhibiting an interesting 35-regular normal non-CI-graph for $Z_{2}^{6}$ that appears to be sporadic. We note in passing that its complement is the unique smallest non-CIgraph for an elementary abelian 2-group, as it is known that $Z_{2}^{k}$ is a CI-group for $k \leq 5$, and the smallest previously known non-CI-graph for $Z_{2}^{6}$ is a 31-regular example complementary to one given by Nowitz [6].

\section{Hadamard graphs}

The Hadamard graph $H(n)$ has the $2 n \pm 1$ vectors of length $n$ as its vertices, where two vertices are adjacent if and only if they are orthogonal. This graph has previously been studied in several contexts (see Ito [3, 4] and Frankl [1]), with one of the more surprising being the fact that determining its chromatic number has applications in quantum computing. The graph $H(8)$ has 256 vertices, and is disconnected with two components, with the vectors with an even (respectively, odd) number of ' -1 's forming the two components. It is easy to see that $x \cdot y=0$ if and only if $x \cdot(-y)=(-x) \cdot y=(-x) \cdot(-y)=0$, and therefore each component of $H(8)$ is a lexicographic product $X\left[2 K_{1}\right]$, where $X$ is a 64-vertex graph. Each of the vertices of $X$ consists of a pair $\{x,-x\}$ of vectors, where $x$ has an even number of ' -1 's. We will identify such a vertex with the vector from $\{x,-x\}$ containing the fewer ' -1 's, or the vector with first coordinate $x_{1}=1$ if $x$ and $-x$ both contain four ' -1 's. The valency of $H(8)$ is $\left(\begin{array}{l}8 \\ 4\end{array}\right)=70$ and so $X$ has valency 35 . The following series of lemmas describes the properties of the 35-valent graph $X$, with respect to the special vertex $\alpha=(1,1,1,1,1,1,1,1)$.

LEMMA 2. The neighbours of $\alpha$ are the 35 vectors $x$ with four ' -1 's and $x_{1}=1$, and the vertices at distance two from $\alpha$ are the 28 vectors with two ' -1 's.

LEMMA 3. The graph $X$ is a $(64,35,18,20)$ strongly regular graph. 
PRoOF OF LEMma 3. Consider two adjacent vertices, which without loss of generality may be taken to be $(1,1,1,1,1,1,1,1)$ and $(-1,-1,-1,-1,1,1,1,1)$. A vector is mutually orthogonal to these if and only if it has two ' -1 's in the first four coordinates, and two ' -1 's in the last four coordinates. There are $36=\left(\begin{array}{l}4 \\ 2\end{array}\right) \times\left(\begin{array}{l}4 \\ 2\end{array}\right)$ such vectors, but, as we have counted each vector and its negative, these correspond to 18 distinct vertices. The common neighbours of two nonadjacent vertices, say $(1,1,1,1,1,1,1,1)$ and $(-1,-1,1,1,1,1,1,1)$, must have one ' -1 ' in the first two coordinates, and three ' -1 's in the last six positions. There are $2 \times\left(\begin{array}{l}6 \\ 2\end{array}\right)=40 \mathrm{such}$ vectors, corresponding to 20 distinct vertices.

LEMMA 4. The full automorphism group of $X$ is equal to the semidirect product

$$
Z_{2}^{6} \rtimes \operatorname{Sym}(8) .
$$

PROOF. Let [8] denote the set $\{1, \ldots, 8\}$ and, for $s \subseteq[8]$, let $\rho_{s}$ denote the operation on $Z_{2}^{8}$ defined by negating the components in the coordinate positions in $s$. It is easy to see that $\rho_{s}$ is an involution and that

$$
\rho_{s} \rho_{t}=\rho_{t} \rho_{s}=\rho_{s \oplus t}
$$

(where $\oplus$ is the symmetric difference operator on subsets of [8]). If $s$ has even cardinality, then $\rho_{s}$ induces an automorphism of $X$, which we shall also denote by $\rho_{s}$, noting that as automorphisms $\rho_{s}=\rho_{t}$ if and only if $s=t$ or $s=t \oplus$ [8]. Therefore, there are $2^{6}$ distinct automorphisms $\rho_{s}$, each taking $\alpha$ to a different vertex, so the collection of all such automorphisms forms a regular subgroup of $\operatorname{Aut}(X)$ isomorphic to $Z_{2}^{6}$. There are 8 ! permutations of the eight coordinate positions, each of which induces an automorphism of $X$, with no two permutations inducing the same automorphism. If we interpret the ordered pair $\left(\sigma, \rho_{s}\right)$ as meaning 'permute coordinates according to $\sigma$, and then apply $\rho_{s}$ ', it follows directly from the definitions that

$$
\left(\sigma, \rho_{s}\right)\left(\tau, \rho_{t}\right)=\left(\sigma \tau, \rho_{s} \tau \rho_{t}\right)
$$

and therefore $\operatorname{Aut}(X)$ contains the semidirect product $Z_{2}^{6} \rtimes \operatorname{Sym}(8)$. The vertices at distance two from $\alpha$ induce a subgraph isomorphic to the complement of the line graph of $K_{8}$, which has full automorphism group isomorphic to $\operatorname{Sym}(8)$. Any automorphism of $X$ fixing this subgraph is necessarily the identity, and so the stabilizer of $\alpha$ has order 8!. Therefore we have identified the full automorphism group of $X$.

COROLlary 5. The graph $X$ is a normal Cayley graph for $Z_{2}^{6}$.

PROOF. The automorphisms of the form $\rho_{s}$ form a normal subgroup of $\operatorname{Aut}(X)$.

THEOREM 6. The graph $X$ is a non-CI-graph.

PROOF. We will exhibit another regular subgroup of $\operatorname{Aut}(X)$ isomorphic to $Z_{2}^{6}$. 
Up to isomorphism, the graph $K_{8}$ has a unique 1-factorization with the additional property that the union of any two 1 -factors is the union of two 4-cycles. From each 1-factor in this 1-factorization, form a permutation of [8] whose cycles correspond to the edges of the 1-factor, yielding the following seven permutations:

$$
\begin{array}{ll}
h_{2}=(12)(34)(56)(78), & h_{6}=(16)(25)(38)(47), \\
h_{3}=(13)(24)(57)(68), & h_{7}=(17)(28)(35)(46), \\
h_{4}=(14)(23)(58)(67), & h_{8}=(18)(27)(36)(45) . \\
h_{5}=(15)(26)(37)(48), &
\end{array}
$$

The special property of the 1-factorization ensures that these permutations commute, and in fact they comprise all the nonidentity elements of a subgroup of $\operatorname{Sym}(8)$ isomorphic to $Z_{2}^{3}$. Every 4-subset of [8] determines a subgraph $K_{4}$ with six edges. This $K_{4}$ either contains two edges in each of three distinct 1-factors, $h_{i}, h_{j}, h_{k}$, where $h_{i} h_{j}=h_{k}$, or no two edges belong to the same 1-factor. There are 14 different 4-subsets in the former category, and taking one from each complementary pair, we get the following seven 4-sets:

$$
\begin{array}{llll}
\{1,2,3,4\}, & \{1,2,5,6\}, & \{1,2,7,8\}, \quad\{1,3,5,7\}, \\
\{1,3,6,8\}, & \{1,4,5,8\}, & \{1,4,6,7\} . &
\end{array}
$$

These sets have the property that the intersection of any two of them has cardinality two, and the intersection of any set with the complement of another also has cardinality two. Let $A$ and $B$ be the following sets of automorphisms, where $e$ is the identity element.

\begin{tabular}{ll}
\hline \multicolumn{1}{c}{$A$} & \multicolumn{1}{c}{$B$} \\
\hline$\left(e, \rho_{\emptyset}\right)$ & $\left(e, \rho_{\emptyset}\right)$ \\
$\left(h_{2}, \rho_{\{1,2\}}\right)$ & $\left(e, \rho_{\{1,2,3,4\}}\right)$ \\
$\left(h_{3}, \rho_{\{1,3\}}\right)$ & $\left(e, \rho_{\{1,2,5,6\}}\right)$ \\
$\left(h_{4}, \rho_{\{1,4\}}\right)$ & $\left(e, \rho_{\{1,2,7,8\}}\right)$ \\
$\left(h_{5}, \rho_{\{1,5\}}\right)$ & $\left(e, \rho_{\{1,3,5,7\}}\right)$ \\
$\left(h_{6}, \rho_{\{1,6\}}\right)$ & $\left(e, \rho_{\{1,3,6,8\}}\right)$ \\
$\left(h_{7}, \rho_{\{1,7\}}\right)$ & $\left(e, \rho_{\{1,4,5,8\}}\right)$ \\
$\left(h_{8}, \rho_{\{1,8\}}\right)$ & $\left(e, \rho_{\{1,4,6,7\}}\right)$ \\
\hline
\end{tabular}

Now for $x, y \in[8]$,

$$
x^{h_{y}}=1^{h_{x} h_{y}}
$$

and

$$
\left(h_{x}, \rho_{\{1, x\}}\right)\left(h_{y}, \rho_{\{1, y\}}\right)=\left(h_{x} h_{y}, \rho_{\left\{1,1^{h_{x}} h_{y}\right\}}\right),
$$

and so $A$ is a subgroup of $\operatorname{Aut}(X)$ isomorphic to $Z_{2}^{3}$. It is simple to verify that $B$ is a subgroup of $\operatorname{Aut}(X)$, also isomorphic to $Z_{2}^{3}$. Furthermore, if we let $a=\left(\sigma, \rho_{s}\right)$ and $b=\left(e, \rho_{t}\right)$, then

$$
a b=\left(\sigma, \rho_{s \oplus t}\right) \quad \text { and } \quad b a=\left(\sigma, \rho_{t}^{\sigma} \oplus s\right) .
$$


If $t$ contains an orbit of $\sigma$, then it is the union of two orbits of $\sigma$ and hence invariant under $\sigma$. Otherwise $t^{\sigma}=t \oplus[8]$ and, as $s$ contains exactly one element in $t^{\sigma}$, it follows that

$$
s \oplus t=t^{\sigma} \oplus s \oplus[8]
$$

In either case it follows that $a b=b a$, and so $A B=B A$, and as $A \cap B=e, A B$ is a subgroup of $\operatorname{Aut}(X)$ isomorphic to $Z_{2}^{6}$. The product $\left(h_{x}, \rho_{\{1, x\}}\right)\left(e, \rho_{s}\right)$ maps $\alpha$ to the vector with ' -1 's in coordinate positions in $s \oplus\{1, x\}$. If $s \oplus\{1, x\}=t \oplus\{1, y\}$ or $s \oplus\{1, x\}=t \oplus\{1, y\} \oplus[8]$, then $s \oplus t=\{x, y\}$ or $s \oplus t=\{x, y\} \oplus$ [8] respectively, neither of which are possible when $s$ and $t$ are chosen from the 4-subsets associated with the elements of $B$. Thus every element of $A B$ maps $\alpha$ to a different vertex of $X$, and so $A B$ is a regular subgroup of $\operatorname{Aut}(X)$.

\section{References}

[1] P. Frankl, 'Orthogonal vectors in the $n$-dimensional cube and codes with missing distances', Combinatorica 6(3) (1986), 279-285.

[2] C. Godsil and G. Royle, Algebraic Graph Theory, Graduate Texts in Mathematics, 207 (Springer, New York, 2001).

[3] N. Ito, 'Hadamard graphs. I', Graphs Combin. 1(1) (1985), 57-64.

[4] - 'Hadamard graphs. II', Graphs Combin. 1(4) (1985), 331-337.

[5] C. H. Li, 'On isomorphisms of finite Cayley graphs-a survey', Discrete Math. 256(1-2) (2002), 301-334.

[6] L. A. Nowitz, 'A non-Cayley-invariant Cayley graph of the elementary abelian group of order 64', Discrete Math. 110(1-3) (1992), 223-228.

GORDON F. ROYLE, School of Mathematics and Statistics, University of Western Australia, Nedlands, WA 6009, Australia

e-mail: gordon@maths.uwa.edu.au 